\title{
Acquisition of the auditory sameldifferent task in a rhesus monkey
}

\author{
MELISSA R. SHYAN, ANTHONY A. WRIGHT, ROBERT G. COOK, and MASAKO JTSUMORI \\ University of Texas Health Science Center, Houston, Texas
}

\begin{abstract}
A rhesus monkey was trained successfully on an auditory same/different task. The monkey showed significant savings in acquisition when transferred to different sound sequences (from 1,480 trials at first acquisition to 25 trials at later transfer). Factors that may have been important in this acquisition were the use of a large stimulus set size (38 trial-unique sounds), sound and response localization cuing, tactual cuing, differential reinforcement of different responding, and early sound localization accompanied by gradual fading. The probe (second) sound was initially presented only through the correct side speaker, right for same sound, left for different sound. After the monkey learned to touch the speaker from which the sound emanated, the sound level coming from the incorrect speaker was gradually increased to full intensity. Only 30 trials at full intensity on both probe speakers were needed before the criterion $(80 \%$ correct responding) was reached. The monkey was then transferred to a new sequence of sounds. Ten sequences were presented in all (including six with novel stimuli). Only the first four sequences required sound localization fading; all others used only full-intensity sound. In all cases, responding reached $80 \%$ correct or better, demonstrating good acquisition of the auditory same/different task.
\end{abstract}

Over the years many attempts have been made (D'Amato \& Colombo, 1985; D'Amato \& Salmon, 1982, 1984a; Dewson \& Cowey, 1969; Dewson, Wertheim, \& Lynch, 1968; Thompson, 1980, 1981; Wegener, 1964) to train monkeys to discriminate auditory stimuli. Monkeys seem not to be able to learn auditory discriminations (e.g., Thompson, 1980, 1981; Wegener, 1964), or to learn them only with great difficulty (D'Amato \& Salmon, 1982, 1984a). D'Amato and Colombo (1985) recently made some progress in that $\mathbf{4}$ of $\mathbf{8}$ monkeys trained showed acquisition of a matching task and showed moderate transfer. The 4 monkeys that showed acquisition took an average of 2,600 trials; however, the other 4 monkeys never acquired the task. This difficulty in training auditory discrimination in monkeys is surprising, because visual discrimination is readily learned (e.g., D'Amato, Salmon, $\&$ Colombo, 1985). Because similar problems occur with dolphins, which learn auditory discriminations well but have great difficulty in acquiring visual tasks (e.g., Herman, 1980), Thompson (1981) suggested that perhaps cognitive processes are sense-specific in some species and that monkeys are cognitively unequipped to learn discriminative tasks with auditory stimuli. ${ }^{1}$ Yet monkeys clearly discriminate sounds in the wild.

This research was funded by National Institute of Mental Health Grant MH-35202 to Anthony A. Wright and by National Institute of Health Institutional Training Grant EY-07024. Reprint requests may be sent to Melissa R. Shyan, Sensory Sciences Center, 6420 Lamar Fleming Blvd., SPI-316, University of Texas Health Science Center at Houston, Houston, TX 77030. Masako Jitsumori is at the Department of Psychology, Chiba University, Yayoi-cho, Chiba-shi, Japan, and was a visiting professor during this project. Robert G. Cook is now at the Department of Psychology, Tufts University, Medford, MA.
Several procedures in some of these previous experiments may have contributed to the monkeys' difficulty in learning the auditory discriminations. First, all of these experiments used small stimulus sets (from two to six sounds) during training. Thompson and Herman (1977; auditory discrimination in dolphins), Wright, Sands, and Urcuioli (1986; visual discrimination in monkeys, humans, and pigeons), and Worsham (1975; visual matching in monkeys) reported that small stimulus sets impede discrimination task acquisition. The animal is unable to temporally differentiate which continually repeated stimulus was most recently presented as the sample (buildup of proactive interference). They found that large stimulus sets and trial-unique sessions (wherein each stimulus is used only once per session) produced rapid acquisition and high performance rates.

Second, several studies presented different mediating stimuli to aid in acquisition. D'Amato and Salmon (1984b), Salmon (1984), and A. A. Wright and R. G. Cook (personal communication, September 5, 1985) presented visual mediating stimuli. Monkeys learn visual tasks so well that pairing auditory and visual stimuli seemed an appropriate way to transfer the concept from visual to auditory modes. In all cases, the visual stimuli completely blocked learning about the auditory stimuli. Indeed, D'Amato and Colombo (1985) felt that the failure of 4 monkeys to acquire was due, in part, to the monkeys' having had prior experience on visual tasks. They suggested that any visual stimulation at all (i.e., from a houselight or lighted response keys) interfered with learning. Sound localization has also been used as a mediating stimulus (Brown, Beecher, Moody, \& Stebbins, 1979; Downey \& Harrison, 1975; Harrison, Downey, Iverson, \& Pratt, 1977). These studies, in which simple "follow- 
the-sound" responding (press the response key near the sound) was trained, did not study auditory discrimination but did produce auditory control of behavior. Some of D'Amato and Colombo's (1985) success in obtaining acquisition and transfer may have been due to their having response levers located adjacent to and below the comparison speakers.

The present experiment incorporated several of the above features and several new features to teach an auditory sameldifferent task. First, a large set of 38 trialunique stimuli was used in training sessions. Second, these were complex stimuli made up of both natural sounds from the monkey's home environment and man-made sounds. Harrison (1980) and Petersen (1982) indicated that use of natural stimuli during training increases the likelihood of success. Previous studies used simple stimuli (pure tones, white noise, simple tunes) and ignored the use of natural environmental sounds. Third, sound localization was used as a mediating stimulus. Speakers were placed in distinctly separate locations within the testing chamber. Touch-screens were used as response manipulanda. These screens were placed directly in front of each speaker, allowing the monkey to "touch the sound" and creating total localization of stimulus and response manipulanda. Fourth, the screens vibrated from sounds emitted, providing additional tactual cuing.

The monkey was first taught to touch the screen in front of whichever speaker was emitting sound. Sample stimuli came from a centrally located speaker. Probes that matched the sample came from the right speaker; probes that differed from the sample came from the left speaker. Gradually, sound intensity on the incorrect probe speaker was increased from zero (no sound) until it equaled the intensity of sound on the correct probe speaker. Thus, sound intensity was manipulated across probe speakers until the only way the monkey could respond correctly was to discriminate identical from nonidentical sounds. One additional factor was introduced: differential reinforcement for correct same and different trials. Denny (1986), Peterson, Wheeler, and Armstrong (1978), Peterson, Wheeler, and Trapoid (1980), and others reported that acquisition of discrimination tasks is facilitated by differential reinforcement.

\section{METHOD}

\section{Subject}

The subject was a 3-year-old male rhesus monkey (Macaca mulatta) named BW. This monkey had been the subject in an earlier study, which had attempted to train the auditory sameldifferent task via visual mediating stimuli paired with auditory stimuli (A. A. Wright and R. G. Cook, personal communication, September 5,1985 ). In that study, the monkey was given 17,376 trials of auditory stimuli alone and then 6,634 trials of visual stimuli paired with auditory stimuli in a same/different paradigm; he failed to achieve higher than chance correct responding.

\section{Stimuli}

Thirty-eight sounds were used for acquisition and training. Two types of acoustic stimuli were used: 20 natural sounds from the monkey's home cage environment (e.g., monkey vocalizations, cage rattles) and 18 manmade sounds (e.g., buzz saw, bell ringing). These stimuli were recorded from the monkey's home cage environment and from 13 sound effects records (Elektra Records).

\section{Apparatus}

The testing cage $(63 \times 63 \times 95 \mathrm{~cm})$ was located within an inhouseconstructed sound-deadening chamber. This chamber was covered by a layer of soundproof acoustic tile, cardboard egg cartons, and 5-cmthick foam padding. Three Beovox C40 (Bang \& Olufsen) speakers were used, a sample-sound speaker centrally located at the front of the testing cage ( $32 \mathrm{~cm}$ from the cage center and $31 \mathrm{~cm}$ from the cage floor), and two probe-sound speakers located at the back on either side of the testing chamber (each $32 \mathrm{~cm}$ from the cage center and $45 \mathrm{~cm}$ from the cage floor). The cage was modified by removing one vertical bar from in front of each speaker to provide adequate space $(8 \times 20 \mathrm{~cm})$ for touch responding (see below). Sounds were presented on TDK metal bias cassette tape via two computer-controlled (Cromemco Z-2D) random-access Beocord 800 tape recorders (Bang \& Olufsen). A computer-controlled relay selected which probe speaker was accessed. A manually controlled variable decibel level control (Realistic Model AT-50H Violet) attached to this relay varied the intensity of sound on the incorrect probe speaker from zero (no sound) until it equaled the intensity of sound on the correct probe speaker. At completely unequal intensities (sound only from the correct speaker), sound measured $51 \mathrm{~dB}$. At completely equal intensities, the sound level from the correct speaker was $55 \mathrm{~dB}$ and that from the incorrect speaker was $53 \mathrm{~dB}$, with a combined intensity of $59 \mathrm{~dB}^{2}$ Intensity on the sample speaker was $55 \mathrm{~dB}$. All measurements were from the center of the testing cage, were averaged over all 38 stimuli used in the experiment, and were measured by a Bruel \& Kjaer precision sound level meter (Type 2215) using the A weighting network.

Tactual cuing was provided by requiring touch responses (touch the screen from which the sound was emitted) to inhouse-designed vibrating touch screens, which were located $2 \mathrm{~cm}$ in front of each speaker, $2 \mathrm{~cm}$ behind the cage wall for the probe speakers, and $5 \mathrm{~cm}$ behind the cage wall for the sample speaker. Wooden houses were fitted tightly around the front and sides of each speaker to increase directionality of the sounds and to hold the $8.5-\mathrm{cm}$-diameter touch screens in the front panel. These touch screens registered the monkey's response via a 74123 TTL integrated circuit. A computer (Cromemco Z-2D) ran each trial, controlled both tape recorders, timed and registered each response, determined whether it was correct or incorrect, and dispensed differential reinforcers or time-outs.

Correct same trials produced 1-gm banana pellets. Correct different trials produced $3 \mathrm{ml}$ of Tang orange drink. Pellet and drink dispensers were located at the back of the cage behind their respective speakers, so that the monkey had to move past the correct speaker to reach the reinforcer.

A dim houselight centrally located in the ceiling was used until the monkey learned the locations of manipulanda and reinforcers (100 trials); then it was turned off to prevent interference from visual cuing.

\section{Procedure}

The first 30 sessions (1,500 trials) of training were an attempt to autoshape the touch response. Sounds were randomly played from either probe speaker for $13 \mathrm{sec}$, after which a banana pellet (for right speaker sounds) or Tang (for left speaker sounds) was dispensed. Although the monkey learned to move to the speaker from which the sound emanated, he did not learn the touch response. Mashed banana was then rubbed on each touch screen to encourage physical contact, after which the monkey acquired the touch response in $\mathbf{3 5 0}$ trials.

He was then required to "follow the sound." A sample sound was presented on the center speaker for $8 \mathrm{sec}$. If the monkey touched the center speaker during this time, the sample sound went off and a probe sound played on one of the two side speakers. If the monkey failed to touch the center speaker at the appropriate time, a 45-sec abort/timeout occurred. A probe sound played on the right speaker when the probe sound matched the sample. A probe sound played on the left speaker when the probe sound did not match the sample. Thus touch-right became the correct response for same trials and touch-left for different trials.

After the monkey followed the sound consistently (which required 80 trials), the sameldifferent task began. The probe sound was gradu- 
ally increased on the incorrect speaker until the monkey was responding correctly with equal amounts of sound out of both probe speakers. The intensity on the incorrect probe speaker was increased as rapidly as possible while the monkey maintained reasonably good performance. If performance deteriorated (two errors in a row at a given intensity), the intensity from the incorrect speaker was reduced until performance improved again. On the first acquisition sequence, the monkey required 1,464 trials (65 sessions) to reach accurate responding at equal intensities (see Note 2). In this fashion the monkey was trained to respond correctly, that is, to make sameldifferent judgments, as localization and tactual cues were made constant across probe speakers.

An intertrial interval of $25 \mathrm{sec}$ was used (including the $12 \mathrm{sec}$ needed for the tape recorders to move to their new locations). Both sample and probe sounds had 2.5-sec fixed-interval observing response requirements to increase the likelihood that the monkey would attend to each sound. Each sound lasted a maximum of $13 \mathrm{sec}$ (including the fixed interval) and the monkey responded at any time after the fixed-interval requirement had been met. A . 75-sec probe delay was used initially, but this was reduced to $0 \mathrm{sec}$, which seemed to prevent anticipatory responding. If an error was made or no response occurred, the sound stopped and an abort/time-out period of $45 \mathrm{sec}$ followed.

Four different training sequences used the same set of 38 sounds (38 for two sequences within 13 different and 12 same trials, 37 for two sequences with 12 different and 13 same trials, counterbalanced across sequences) to produce 25 trial-unique session trials. The order of same and different trials and the pairing of sounds was different for each sequence, and each sequence was presented until a criterion of at least $80 \%$ correct responding at equal intensities was achieved. Once criterion was reached, the monkey was transferred to the next sequence at equal intensities, but was trained to criterion on this new sequence by variation of intensity on the incorrect speaker.

\section{RESULTS AND DISCUSSION}

Number of trials to criterion for each sequence and for later novel-sound transfer sequences (presented for acquisition comparison purposes only) are presented in
Figure 1 . The monkey took 1,480 trials to reach criterion for the first sequence (Condition A). This number is roughly equivalent to that obtained by Dewson et al. (1968) with a go/no-go procedure using only two sounds as stimuli. The next two sequences (Conditions B and C) showed a dramatic decrease in the number of trials to criterion (Condition B, 650 trials; Condition C, 250 trials). For the fourth sequence of the same 38-stimulus set (Condition $\mathrm{D}+\mathrm{E}$ ), the monkey took 275 trials to reach criterion, but this number is misleading. After 250 trials (Condition D) it was noticed that the monkey anticipated the probe onset; that is, he would guess as to which side, and would begin making responses even before the probe sound came on. The .75-sec probe delay was eliminated and the monkey immediately reached criterion (Condition E, 25 trials, or one session).

Sequences F, G, H, I, J, and K contained some novel as well as some familiar stimuli (an increase in the stimulus set size). These sequences were presented only with full intensities on both probe speakers. On Sequence F, which contained 6 novel and 19 familiar sound trials, the monkey reached criterion in 50 trials. Sequence $\mathbf{G}$ contained 12 novel and 13 familiar sound trials, and the monkey reached criterion in one session (25 trials). Sequence $H$, which contained 25 novel sound trials, was presented for 150 trials, but was not run to criterion, as it seemed that the large number of novel stimuli (38) produced undue strain on the monkey at this point in training. Sequence I contained 12 novel and 13 familiar sound trials, and was also not run to criterion. Sequence $J$ contained 12 novel and 13 familiar sound trials, and crite-

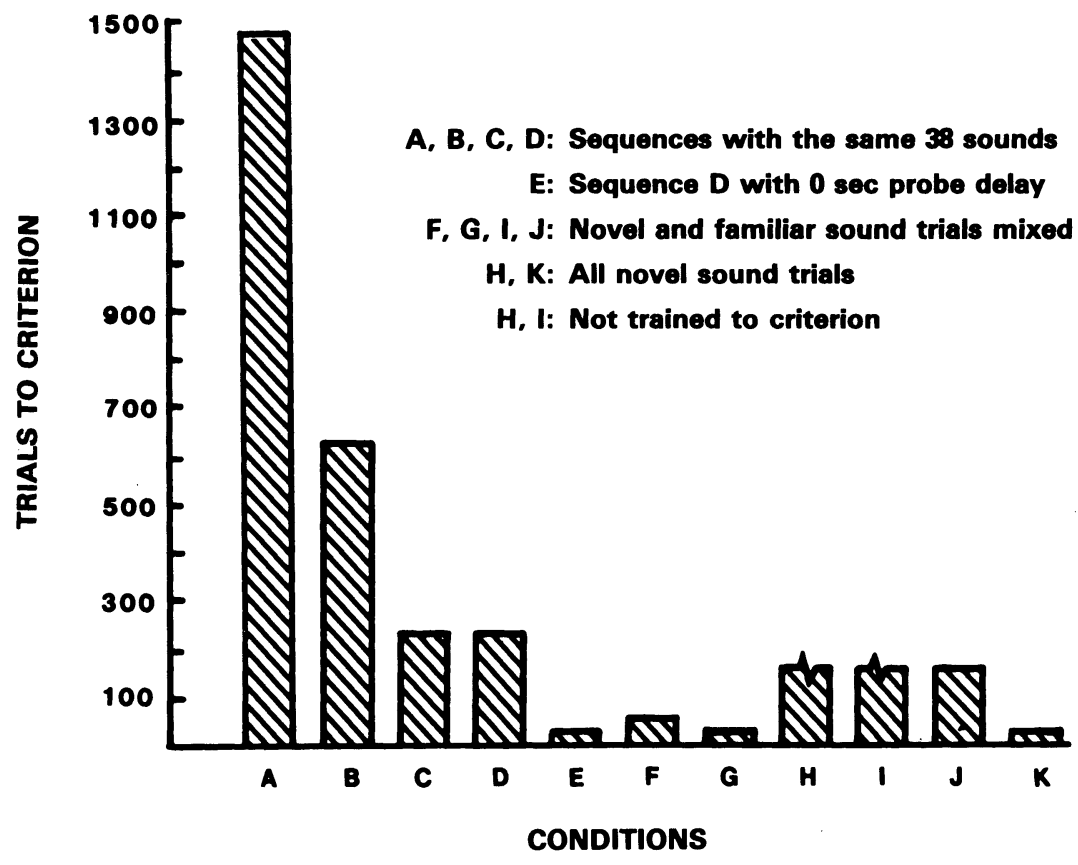

Figure 1. The number of trials to criterion is presented for each sound sequence, including both familiar and novel sound sequences. The number of trials decreased dramatically after initial training, indicating that the monkey was completely successful in acquiring the sameldifferent task. 
rion was reached in 150 trials. On Sequence K, which contained 25 novel sound trials, the monkey required only one session for criterion level performance. ${ }^{3}$

These results show complete success in training a monkey to acquire the sameldifferent task with auditory stimuli. Initial training required nearly as many trials as had been required in some previous studies (e.g., Dewson et al., 1968), slightly more than some (Thompson, 1980, 1981) and substantially fewer than others (D'Amato \& Colombo, 1985; Dewson \& Cowey, 1969; Wegener, 1964). Substantially less training was required on different sequences using the same 38 trial-unique sounds, and later on different sequences introducing novel sounds, than has been required in any other published study. Our work shows that it is premature to suggest that monkeys cannot learn auditory discrimination tasks. With the combined use of localization and tactual cuing, the intensity fading procedure, and differential reinforcement of different responses, acquisition with auditory stimuli can be comparable to acquisition with visual stimuli. The present study also shows that, although D'Amato and Colombo (1985) reported that visually contaminated subjects did poorly on auditory tasks, this is not the rule for all situations. The present subject had extensive experience on a visually mediated auditory learning task, with responding at chance ( $49 \%$ correct), and yet performed quite well on the present auditory task ( $80 \%$ correct or better). These findings open up the possibility of testing auditory list memory, tune and melody perception, cross-modal perception, and cognitive and neurophysiological animal models of human auditory memory.

\section{REFERENCES}

Brown, C. H., Beecher, M. D., Moody, D. B., \& Stebins, W. C. (1979). Locatability of vocal signals in old world monkeys: Design features for the communication of position. Journal of Comparative \& Physiological Psychology, 93, 806-819.

D'Amato, M. R., \& CoLomBo, M. (1985). Auditory matching-to-sample in monkeys (Cebus apella). Unpublished manuscript.

D'Amato, M. R., \& SAlmon, D. P. (1982). Tune discrimination in monkeys (Cebus apella) and rats. Animal Learning \& Behavior, 10, 126-134.

D'Amato, M. R., \& Salmon, D. P. (1984a). Cognitive processes in cebus monkeys. In H. L. Roitblat, T. G. Bever, \& H. S. Terrace (Eds.), Animal cognition (pp. 149-168). Hillsdale, NJ: Erlbaum.

D'Amato, M. R., \& Salmon, D. P. (1984b). Processing and retention of complex auditory stimuli in monkeys (Cebus apella). Canadian Journal of Psychology, 38, 237-255.

D'Amato, M. R., SAlmon, D. P., \& Colombo, M. (1985). Extent and limits of the matching concept in monkeys (Cebus apella). Journal of Experimental Psychology: Animal Behavior Processes, 2, 176-196.

DENNY, M. R. (1986). "Retention" of S-R in the midst of the cognitive invasion. In D. F. Kendrick, M. Rilling, \& M. R. Denny (Eds.), Theories of animal memory (pp. 101-128). Hillsdale, NJ: Erlbaum.

Dewson, J. H., III, \& CoweY, A. (1969). Discrimination of auditory sequences by monkeys. Nature, 222, 695-697.

Dewson, J. H., III, Wertheim, G. A., Lynch, J. C. (1968). ACquisition of successive auditory discrimination in makeup. Journal of the Acoustical Society of America, 43, 162-163.

Downey, P., \& HARRISON, J. M. (1975). Control of responding by sound location in monkeys: Rapid acquisition in darkness. Journal of the Experimental Analysis of Behavior, 23, 265-276.
HARRISON, J. M. (1980). Naturalistic considerations in the study of discrimination. In (n.ed.) nt, 319-335.

Harrison, J. M., Downey, P., Iverson, S. D., \& Pratt, S. R. (1977). Control of responding by location of auditory stimuli: Adjacency of sound and response. Journal of the Experimental Analysis of Behavior, 28, 243-251.

Herman, L. M. (1980). Cognitive characteristics of dolphins. In L. M. Herman (Ed.), Cetacean behavior: Mechanisms and functions. New York: Wiley Interscience.

Petersen, M. R. (1982). The peception of species-specific vocalizations by primates: A conceptual framework. In C. T. Snowdon, C. H. Brown, \& M. R. Petersen (Eds.), Primate communication (pp. 171211). New York: Cambridge University Press.

Peterson, G. B., Wheeler, R. L., \& Armstrong, G. D. (1978). Expectancies as mediating the differential-reward conditional discrimination performance of pigeons. Animal Learning \& Behavior, 6, 276-285.

Peterson, G. B., Wheeler, R. L., \& Trapoid, M. A. (1980). Enhancement of pigeon's conditional discrimination performance by expectancy of reward. Animal Learning \& Behavior, 8, 22-30.

SALmon, D. P. (1984). An investigation of modality specificity in the cognitive processes of monkeys (Cebus apella). Dissertation Abstracts International, 45, 706.

Thompson, R. K. R. (1980). Auditory cued reversal and matching-tosample learning by rhesus monkeys. Antropologia Contemporanea, 3, 284-292.

THOMPSON, R. K. R. (1981). Follow-up to the auditory matching by a monkey paper. Unpublished manuscript.

Thompson, R. K. R., Herman, L. M. (1977). Memory for lists of sounds by the bottle-nosed dolphin: Convergence of memory processes with humans? Science, 195, 501-503.

WEGENER, J. G. (1964). Auditory discrimination behavior of normal monkeys. Journal of Auditory Research, 4, 81-106.

Worsham, R. W. (1975). Temporal discrimination factors in the delayed matching-to-sample task in monkeys. Animal Learning \& Behavior, 3, 93-97.

Wright, A. A., Sands, S. F., \& Urcuioli, P. J. (1986). Proactive interference in animal memory. In D. F. Kendrick, M. Rilling, \& M. R. Denny (Eds.), Theories of animal memory (pp. 101-128). Hillsdale, NJ: Erlbaum.

\section{NOTES}

1. L. M. Herman (personal communication, March 2, 1985) has had recent success in training discrimination of three-dimensional objects through the use of two mediating stimuli: motion and auditory pairing. A dolphin trained to comprehend an acoustic language was taught the matching-to-sample task by presenting the sample object with stereotyped motion, playing its acoustic name, and then presenting two stationary probe-object choices. Both motion and name were gradually faded. The dolphin demonstrated $80 \%$ or better correct responding, including responses to unnamed novel objects.

2. Once the monkey reached accurate responding at equal intensities, the relay that selected the correct probe speaker was locked so that it no longer functioned. The slightly louder $(55 \mathrm{~dB})$ speaker was always the left (different) speaker and the slightly softer $(53 \mathrm{~dB})$ speaker was always the right (same) speaker during equal intensity trials. Thus the slight difference in intensities found between the correct and incorrect probe speakers at equal intensities was controlled in that this difference was kept constant across same and different trials and could not serve as a response cue.

3. It appears that the additional two months experience between the first completely novel sequence, Sequence $H$, and the second, Sequence $K$, produced enough learning for correct responding to a large number of novel sounds.

(Manuscript received for publication October 1, 1986.) 